\title{
CHIRAL-SYMMETRY AND STRANGE FOUR-QUARK MATRIX ELEMENTS*
}

\author{
Xiangdong Ji \\ Center for Theoretical Physics \\ Laboratory for Nuclear Science \\ and Department of Physics \\ Massachusetts Institute of Technology \\ Cambridge, Massachusetts 02139
}

\begin{abstract}
We consider the matrix elements of the left-handed flavor-conserving four-quark operators in the nucleon and pion states. Using chiral symmetry, we derive relationships among these matrix elements. We argue that the $\Delta I=1 / 2$ rule of hyperon and kaon non-leptonic weak decay implies possible large strange-quark content in the nucleon and pion.
\end{abstract}

Submitted to: Physics Letters B

CTP\#2188

hep-ph/9307234

February 1993

* This work is supported in part by funds provided by the U.S. Department of Energy (D.O.E.) under contract \#DE-AC02-76ER03069. 
The nucleon's strange content has received considerable attention since the EMC measurement of the proton's spin structure function $g_{1}(x) .{ }^{1}$ Many more deep-inelastic and (quasi)elastic experiments are now in running or have been proposed to make further measurement of strange-quark matrix elements. ${ }^{2}$ Study of the nucleon's non-valence degrees of freedom helps us to understand better the role played by the sea of Quantum Chromodynamics (QCD). It also provides clues for more realistic model-building and for finding better approximations to solve QCD.

In this letter, we discuss the strange four-quark matrix elements in the nucleon and pion states. Our discussion is motivated by a recent paper by Kaplan, ${ }^{3}$ who showed that the wellknown $\Delta I=1 / 2$ rule of hyperon non-leptonic weak decay implies large strange four-quark matrix element, $\left\langle P\left|\bar{u}_{L} \gamma_{\mu} s_{L} \bar{s}_{L} \gamma^{\mu} d_{L}\right| N\right\rangle$. Our goal here is to elaborate on his result from the relationships among four-quark matrix elements implied by chiral symmetry, and to show that the vanishing strange-quark matrix elements are incompatible with the $\Delta I=1 / 2$ rule. Then we extend our discussion to the pion and argue that accommodation of both the $\Delta I=1 / 2$ rule and vanishing strange-quark matrix elements requires a drastically different valence quark model from what we have constructed. If there is a trail of pion large strange content here, this may be the first that the pion has an intricate flavor structure.

Let us consider the following tensor of four-quark operators,

$$
T_{j l}^{i k}=\frac{1}{4} \bar{q}_{i} \gamma^{\mu}\left(1-\gamma_{5}\right) q_{j} \bar{q}_{k} \gamma_{\mu}\left(1-\gamma_{5}\right) q_{l},
$$

which will be denoted by $\left(\bar{q}_{i} q_{j}\right)\left(\bar{q}_{k} q_{l}\right)$, or simply $\bar{q}_{i} q_{j} \bar{q}_{k} q_{l}$. The indices $i, j, k, l$ run through light-quark flavors $u, d$, and $s$. Color indices, unless specified explicitly, are coupled to singlet in the quark pairs $(i, j)$ and $(k, l)$. The tensor is symmetric under simultaneous exchange of $i$ and $k$, and $j$ and $l$, and thus symmetrizing $i$ and $k$ and anti-symmetrizing $j$ and $l$, or vice versa, yield a null result. We have therefore a total of $3^{4}-3 \times 6 \times 2=45$ independent tensor components. The symmetrized tensor $T_{(j l)}^{(i k)}$ has $6 \times 6=36$ components and the antisymmetrized tensor $T_{[j l]}^{[i k]}$ has $3 \times \overline{3}=9$ components. The former contains 27,8 , and 1 representations of $S U(3)_{L}$ and the latter 8 and 1 representations.

The construction of the operators belonging to different $S U(3)_{L}$ representations is standard. Here we present the result in order to specify the normalization we adopt. For 27, we subtract away the trace of the symmetric tensor,

$$
\begin{aligned}
\bar{T}_{(j l)}^{(i k)}= & T_{(j l)}^{(i k)}-\frac{1}{5}\left[T_{(m l)}^{(m k)} \delta_{j}^{i}+T_{(m j)}^{(m i)} \delta_{l}^{k}+T_{(m l)}^{(m i)} \delta_{j}^{k}+T_{(m j)}^{(m k)} \delta_{l}^{i}\right] \\
& +\frac{1}{20} T_{(m n)}^{(m n)}\left[\delta_{j}^{i} \delta_{l}^{k}+\delta_{l}^{i} \delta_{j}^{k}\right] .
\end{aligned}
$$

For the symmetric and anti-symmetric octets we define,

$$
O_{S, A}^{a}=\left(\bar{q}_{\alpha} T^{a} q_{\alpha}\right)\left(\bar{q}_{\beta} q_{\beta}\right) \pm\left(\bar{q}_{\alpha} T^{a} q_{\beta}\right)\left(\bar{q}_{\beta} q_{\alpha}\right)
$$


where the Gell-Mann matrices $T^{a}$ are normalized according to $\operatorname{Tr} T^{a} T^{b}=1 / 2 \delta^{a b}$. The repeated $\alpha$ and $\beta$ indices denote summation over color, and the flavor indices are coupled in each bracket implicitly. For symmetric and anti-symmetric singlets we define,

$$
S_{S, A}=\left(\bar{q}_{\alpha} q_{\alpha}\right)\left(\bar{q}_{\beta} q_{\beta}\right) \pm\left(\bar{q}_{\alpha} q_{\beta}\right)\left(\bar{q}_{\beta} q_{\alpha}\right)
$$

Any tensor component in eq. (1) can be decomposed into a sum of operators belong to these five different representations. For diagonal hadron matrix elements, we are interested in operators with no net flavor change. There are nine of them: $\bar{u} u \bar{u} u, \bar{d} d \bar{d} d, \bar{s} s \bar{s} s, \bar{u} u \bar{d} d$, $\bar{u} u \bar{s} s, \bar{d} d \bar{s} s, \bar{u} d \bar{d} u, \bar{u} s \bar{s} u$, and $\bar{d} s \bar{s} d$. Their decomposition into different representations is straightforward,

$$
\begin{gathered}
\bar{u} u \bar{u} u=\bar{T}_{u u}^{u u}+\frac{2}{5} O_{S}^{3}+\frac{2}{5 \sqrt{3}} O_{S}^{8}+\frac{1}{12} S_{S}, \\
\bar{d} d \bar{d} d=\bar{T}_{d d}^{d d}-\frac{2}{5} O_{S}^{3}+\frac{2}{5 \sqrt{3}} O_{S}^{8}+\frac{1}{12} S_{S}, \\
\bar{s} s \bar{s} s=\bar{T}_{s s}^{s s}-\frac{4}{5 \sqrt{3}} O_{S}^{8}+\frac{1}{12} S_{S}, \\
\frac{1}{2}(\bar{u} u \bar{d} d+\bar{u} d \bar{d} u)=\bar{T}_{u d}^{u d}+\frac{1}{5 \sqrt{3}} O_{S}^{8}+\frac{1}{24} S_{S}, \\
\frac{1}{2}(\bar{d} d \bar{s} s+\bar{d} s \bar{s} d)=\bar{T}_{d s}^{d s}-\frac{1}{10} O_{S}^{3}-\frac{1}{10 \sqrt{3}} O_{S}^{8}+\frac{1}{24} S_{S}, \\
\frac{1}{2}(\bar{s} s \bar{u} u+\bar{s} u \bar{u} s)=\bar{T}_{s u}^{s u}+\frac{1}{10} O_{S}^{3}-\frac{1}{10 \sqrt{3}} O_{S}^{8}+\frac{1}{24} S_{S}, \\
\frac{1}{2}(\bar{u} u \bar{d} d-\bar{u} d \bar{d} u)=\frac{1}{\sqrt{3}} O_{A}^{8}+\frac{1}{12} S_{A}, \\
\frac{1}{2}(\bar{d} d \bar{s} s-\bar{d} s \bar{s} d)=-\frac{1}{12} O_{A}^{3}-\frac{1}{2 \sqrt{3}} O_{A}^{8}+\frac{1}{12} S_{A}, \\
\frac{1}{2}(\bar{s} s \bar{u} u-\bar{s} u \bar{u} s)=\frac{1}{12} O_{S}^{3}-\frac{1}{2 \sqrt{3}} O_{A}^{8}+\frac{1}{12} S_{A} \cdot
\end{gathered}
$$

Likewise, we can decompose the $\Delta s=1$ non-leptonic weak decay hamiltonian density,

$$
\mathcal{H}=\frac{G_{F}}{\sqrt{2}} s_{1} c_{1} 4(\bar{d} u)(\bar{u} s)
$$

However, this hamiltonian density is defined at the scale of $M_{W}$, the mass of the $W$ boson. Since we are interested in the non-perturbative part of the four-quark matrix elements, we have to run down the scale using a renormalization group equation. The calculation is standard and a recent reference shows, ${ }^{4}$,

$$
\mathcal{H}(\mu=1 \mathrm{GeV})=\frac{G_{F}}{\sqrt{2}} s_{1} c_{1}\left[2.8 \bar{T}_{u s}^{d u}+0.28 O_{S}^{6+i 7}-3.64 O_{A}^{6+i 7}+0.003 Q_{5}-0.01 Q_{6}\right]
$$


where

$$
\begin{aligned}
& Q_{5}=\bar{d}_{\alpha} \gamma^{\mu}\left(1-\gamma_{5}\right) s_{\alpha} \bar{q}_{\beta} \gamma_{\mu}\left(1+\gamma_{5}\right) q_{\beta}, \\
& Q_{6}=\bar{d}_{\alpha} \gamma^{\mu}\left(1-\gamma_{5}\right) s_{\beta} \bar{q}_{\beta} \gamma_{\mu}\left(1+\gamma_{5}\right) q_{\alpha},
\end{aligned}
$$

are generated from the penguin diagram and are interesting due to their distinct chiral structure.

We now consider the matrix elements of those flavor-conserving operators in the nucleon and pion states. [Since the operators are scale-dependent, we assume to work at the scale of $1 \mathrm{GeV}$.] To do that, we first map these quark operators onto operators containing baryon and meson fields with the same flavor symmetry. We use the non-linear representations for mesons and baryons employed in ref. 5: the Goldstone bosons octet is represented by a $3 \times 3$ matrix,

$$
\Sigma=\exp \left(2 i \pi / f_{\pi}\right)
$$

where

$$
\pi=\frac{1}{\sqrt{2}}\left(\begin{array}{ccc}
\frac{1}{\sqrt{2}} \pi^{0}+\frac{1}{\sqrt{6}} \eta & \pi^{+} & K^{+} \\
\pi^{-} & -\frac{1}{\sqrt{2}} \pi^{0}+\frac{1}{\sqrt{6}} \eta & K^{0} \\
K^{-} & K^{0} & -\frac{2}{\sqrt{6}} \eta
\end{array}\right)
$$

and the baryon octet is represented by

$$
B=\left(\begin{array}{ccc}
\frac{1}{\sqrt{2}} \Sigma^{0}+\frac{1}{\sqrt{6}} \Lambda & \Sigma^{+} & P \\
\Sigma^{-} & -\frac{1}{\sqrt{2}} \Sigma^{0}+\frac{1}{\sqrt{6}} \Lambda & N \\
\Xi^{-} & \Xi^{0} & -\frac{2}{\sqrt{6}} \Lambda
\end{array}\right) .
$$

Under chiral transformation, we have

$$
\begin{aligned}
\Sigma & \rightarrow L \Sigma R^{\dagger}, \\
\xi=\sqrt{\Sigma} & \rightarrow L \xi U^{\dagger}=U \xi R^{\dagger}, \\
B & \rightarrow U B U^{\dagger} .
\end{aligned}
$$

In constructing effective operators, we keep only leading terms in chiral perturbation expansion. For 27, we have

$$
\bar{T}_{(j l)}^{(i k)} \rightarrow a \bar{B}_{(j l)}^{(i k)}
$$

where the tensor $B$ is,

$$
B_{(j l)}^{(i k)}=\left(\xi \bar{B} \xi^{\dagger}\right)_{i}^{j}\left(\xi B \xi^{\dagger}\right)_{k}^{l}
$$

and for 8's,

$$
O_{S, A}^{a} \rightarrow F_{S, A} \operatorname{Tr} \bar{B}\left[\xi^{\dagger} T^{a} \xi, B\right]+D_{S, A} \operatorname{Tr} \bar{B}\left[\xi^{\dagger} T^{a} \xi, B\right]_{+},
$$

and finally for 1's,

$$
S_{S, A} \rightarrow s_{s, a} \operatorname{Tr} \bar{B} B
$$

The seven parameters, $a, F_{S, A}, D_{S, A}$, and $s_{s, a}$ determine all matrix elements of the four-quark operators between the baryon octet states plus an arbitrary number of Goldstone bosons. 
Using the above mapping, we calculate the matrix elements of the flavor-conserving operators between the proton states,

$$
\begin{gathered}
\langle P|\bar{u} u \bar{u} u| P\rangle=-\frac{3}{20} a+\frac{1}{5}\left(F_{S}+D_{S}\right)+\frac{1}{15}\left(3 F_{S}-D_{S}\right)+\frac{s_{s}}{12}, \\
\langle P|\bar{d} d \bar{d} d| P\rangle=\frac{1}{20} a-\frac{1}{5}\left(F_{S}+D_{S}\right)+\frac{1}{15}\left(3 F_{S}-D_{S}\right)+\frac{s_{s}}{12}, \\
\langle P|\bar{s} s \bar{s} s| P\rangle=-\frac{3}{20} a-\frac{2}{15}\left(3 F_{S}-D_{S}\right)+\frac{s_{s}}{12}, \\
\left\langle P\left|\frac{1}{2}(\bar{u} u \bar{d} d+\bar{u} d \bar{d} u)\right| P\right\rangle=-\frac{1}{40} a+\frac{1}{30}\left(3 F_{S}-D_{S}\right)+\frac{s_{s}}{24}, \\
\left\langle P\left|\frac{1}{2}(\bar{d} d \bar{s} s+\bar{d} s \bar{s} d)\right| P\right\rangle=-\frac{1}{40} a-\frac{1}{20}\left(F_{S}+D_{S}\right)-\frac{1}{60}\left(3 F_{S}-D_{S}\right)+\frac{s_{s}}{24}, \\
\left\langle P\left|\frac{1}{2}(\bar{s} s \bar{u} u+\bar{s} u \bar{u} s)\right| P\right\rangle=\frac{7}{40} a+\frac{1}{20}\left(F_{S}+D_{S}\right)-\frac{1}{60}\left(3 F_{S}-D_{S}\right)+\frac{s_{s}}{24}, \\
\left\langle P\left|\frac{1}{2}(\bar{u} u \bar{d} d-\bar{u} d \bar{d} u)\right| P\right\rangle=\frac{1}{6}\left(3 F_{A}-D_{A}\right)+\frac{s_{a}}{12}, \\
\left\langle P\left|\frac{1}{2}(\bar{d} d \bar{s} s-\bar{d} s \bar{s} d)\right| P\right\rangle=-\frac{1}{4}\left(F_{A}+D_{A}\right)-\frac{1}{12}\left(3 F_{A}-D_{A}\right)+\frac{s_{a}}{12}, \\
\left\langle P\left|\frac{1}{2}(\bar{s} s \bar{u} u-\bar{s} u \bar{u} s)\right| P\right\rangle=\frac{1}{4}\left(F_{A}+D_{A}\right)-\frac{1}{12}\left(3 F_{A}-D_{A}\right)+\frac{s_{a}}{12} .
\end{gathered}
$$

The coefficients in front of the invariant parameters are related to the SU(3) Clebsch-Gordon coefficients.

If we assume that five strange quark matrix elements in eq. (17) vanish, we immediately derive five relations among seven invariant parameters,

$$
\begin{aligned}
F_{A} & =-D_{A}=\frac{1}{4} s_{a}, \\
a & =-\frac{1}{3} s_{s}, \\
F_{S} & =\frac{5}{12} s_{s}, \\
D_{S} & =\frac{1}{4} s_{s} .
\end{aligned}
$$

Here we have taken $s_{a}$ and $s_{s}$ as independent. The rest four non-strange matrix elements can be expressed in terms of these two parameters,

$$
\begin{aligned}
\langle P|\bar{u} u \bar{u} u| P\rangle & =\frac{1}{3} s_{s}, \\
\langle P|\bar{d} d \bar{d} d| P\rangle & =0, \\
\left\langle P\left|\frac{1}{2}(\bar{u} u \bar{d} d-\bar{u} d \bar{d} u)\right| P\right\rangle & =\frac{1}{4} s_{a}, \\
\left\langle P\left|\frac{1}{2}(\bar{u} u \bar{d} d+\bar{u} d \bar{d} u)\right| P\right\rangle & =\frac{1}{12} s_{s} .
\end{aligned}
$$


The vanishing of the four- $d$-quark matrix element is a little surprising, but it can be simply interpreted in valence quark models in which there is only one $d$ quark and thus the two-body matrix element must vanish. However, our result is independent of valence quark models and is directly linked to the $\mathrm{SU}(3)$ symmetry and vanishing strange-quark matrix elements. The relation between the four- $u$-matrix element and the $u d$-symmetric matrix element (the last one in eq. (19)) is simply a consequence of isospin symmetry.

We argue, however, that the pattern of four-quark matrix elements shown in eqs. (18) and (19) is inconsistent with the data on $\Delta s=1$ hyperon non-leptonic decay. To show this, we map the hamiltonian in eq. (7) onto an operator with meson and baryon fields and use it to calculate the hyperon decay rates. Here we neglect the contributions of the penguin operators because of their small coefficients (a more careful analysis was made in ref. 3). From fitting to experimental data, ${ }^{6}$ we have

$$
\begin{aligned}
0.07 F_{S}-0.91 F_{A} & =1.4 \eta \frac{m_{\pi}^{2} f_{\pi}}{2 s_{1} c_{1}} \\
0.07 D_{S}-0.91 D_{A} & =-0.58 \eta \frac{m_{\pi}^{2} f_{\pi}}{2 s_{1} c_{1}}
\end{aligned}
$$

and $a$ is negligible. Here $\eta$ is a phase factor, and time-reversal symmetry restricts $\eta$ to \pm 1 . From eq. (18), the size of $a$ restricts $F_{S}$ and $D_{S}$ to be small, i.e., the symmetric octet is also strongly suppressed if all the strange quark matrix elements vanish. Then from eq. (20), we find the ratio between $F_{A}$ and $D_{A}$ is -2.4 , which contradicts with $F_{A} / D_{A}=-1$, which is implied by eq. (18).

Thus some strange matrix elements must be large. From eq. (17) and the fact that $a$ is small, we have,

$$
\begin{gathered}
\frac{1}{5}\left(F_{S}+D_{S}\right)=\langle P|(\bar{u} u-\bar{d} d) \bar{s} s+(\bar{u} s \bar{s} u-\bar{d} s \bar{d} u)| P\rangle \\
\left(F_{A}+D_{A}\right)=\langle P|(\bar{u} u-\bar{d} d) \bar{s} s-(\bar{u} s \bar{s} u-\bar{d} s \bar{d} u)| P\rangle .
\end{gathered}
$$

Combining these with eq. (20), we have,

$$
\langle P|-0.56(\bar{u} u-\bar{d} d) \bar{s} s+1.26(\bar{u} s \bar{s} u-\bar{d} s \bar{s} d)| P\rangle=0.82 \eta \frac{m_{\pi}^{2} f_{\pi}}{2 s_{1} c_{1}}
$$

which is the matrix element obtained by Kaplan, ${ }^{3}$ except for an isospin rotation.

In the rest of the letter, we show that the above line of discussion can be extended to the pion's matrix elements, although some arguments for the conclusion are less tight. In the Goldstone boson sector, we have the following mapping for the four quark matrix elements:

$$
\bar{T}_{(j l)}^{(i k)} \rightarrow a \frac{f_{\pi}^{2}}{m_{\pi}^{2}} \bar{P}_{(j l)}^{(i k)},
$$

where the tensor $P_{(j l)}^{(i k)}$ is defined as,

$$
P_{(j l)}^{(i k)}=\left(\Sigma \partial^{\mu} \Sigma^{\dagger}\right)_{i}^{j}\left(\Sigma \partial_{\mu} \Sigma^{\dagger}\right)_{k}^{l}
$$


and

$$
\begin{aligned}
O_{S, A}^{a} & \rightarrow b_{s, a} \frac{f_{\pi}^{2}}{m_{\pi}^{2}} \operatorname{Tr}\left[T^{a} \partial_{\mu} \Sigma \partial^{\mu} \Sigma^{\dagger}\right] \\
S_{S, A} & \rightarrow s_{s, a} \frac{f_{\pi}^{2}}{m_{\pi}^{2}} \operatorname{Tr}\left[\partial_{\mu} \Sigma \partial^{\mu} \Sigma^{\dagger}\right]
\end{aligned}
$$

where we introduce five invariant parameters, $a, b_{s, a}$, and $s_{s, a}$. Using these, we obtain the following four-quark matrix elements of the pion,

$$
\begin{aligned}
\left\langle\pi^{0}|\bar{u} u \bar{u} u| \pi^{0}\right\rangle & =-\frac{7}{40} a+\frac{1}{30} b_{s}+\frac{1}{12} s_{s}, \\
\left\langle\pi^{0}|\bar{s} s \bar{s} s| \pi^{0}\right\rangle & =-\frac{1}{40} a-\frac{1}{15} b_{s}+\frac{1}{12} s_{s}, \\
\left\langle\pi^{0}\left|\frac{1}{2}[\bar{u} u \bar{d} d+\bar{u} d \bar{d} u]\right| \pi^{0}\right\rangle & =\frac{13}{80} a+\frac{1}{60} b_{s}+\frac{1}{24} s_{s}, \\
\left\langle\pi^{0}\left|\frac{1}{2}[\bar{u} u \bar{s} s+\bar{u} s \bar{s} u]\right| \pi^{0}\right\rangle & =\frac{1}{80} a-\frac{1}{120} b_{s}+\frac{1}{24} s_{s}, \\
\left\langle\pi^{0}\left|\frac{1}{2}[\bar{u} u \bar{d} d-\bar{u} d \bar{d} u]\right| \pi^{0}\right\rangle & =\frac{1}{12} b_{a}+\frac{1}{12} s_{a}, \\
\left\langle\pi^{0}\left|\frac{1}{2}[\bar{u} u \bar{s} s-\bar{u} s \bar{s} u]\right| \pi^{0}\right\rangle & =-\frac{1}{24} b_{a}+\frac{1}{12} s_{a} .
\end{aligned}
$$

The matrix elements of three other operators, $\bar{d} d \bar{d} d, \bar{d} d \bar{s} s$, and $\bar{d} s \bar{s} d$, are redundant because of isospin symmetry.

If we assume all strange-quark matrix elements vanish, we derive three relations among five invariant parameters,

$$
\begin{aligned}
& b_{a}=2 s_{a}, \\
& b_{s}=-a=2 s_{s} .
\end{aligned}
$$

Three non-strange matrix elements are,

$$
\begin{aligned}
\left\langle\pi^{0}|\bar{u} u \bar{u} u| \pi^{0}\right\rangle & =\frac{1}{2} s_{s}, \\
\left\langle\pi^{0}\left|\frac{1}{2}[\bar{u} u \bar{d} d+\bar{u} d \bar{d} u]\right| \pi^{0}\right\rangle & =-\frac{1}{4} s_{s}, \\
\left\langle\pi^{0}\left|\frac{1}{2}[\bar{u} u \bar{d} d-\bar{u} d \bar{d} u]\right| \pi^{0}\right\rangle & =\frac{1}{4} s_{a} .
\end{aligned}
$$

The first two matrix elements are again related by isospin rotation.

We now argue that eqs. (27) and (28) and the $\Delta I=1 / 2$ rule imply either a nonconventional valence quark model or a large strange content in the pion. From the neutral and charged $K$ non-leptonic decay, we can extract the strengths of the 27 and $8,{ }^{5}$

$$
\begin{aligned}
a & =1.4 \times 10^{-5} \eta \mathrm{GeV}^{4} \\
0.07 b_{s}-0.91 b_{a} & =2.14 \times 10^{-4} \eta^{\prime} \mathrm{GeV}^{4}
\end{aligned}
$$


To be more precise, the second equation above shall include the penguin contributions which are potentially important in the meson sector due to the special chiral structure of $Q_{5}$ and $Q_{6} \cdot{ }^{7}$ Since their precise size at $1 \mathrm{GeV}$ is uncertain, we neglect it temporarily. Coupling eq. (29) with eq. (27) we deduce,

$$
\begin{aligned}
& s_{s}=-0.7 \times 10^{-5} \eta \mathrm{GeV}^{4}, \\
& s_{a}=-1.2 \times 10^{-4} \eta^{\prime} \mathrm{GeV}^{4},
\end{aligned}
$$

and so $\left|s_{a} / s_{s}\right| \sim 17$. Or, in terms of a ratio of non-strange matrix elements,

$$
\chi=\frac{\langle\pi|\bar{u} d \bar{d} u| \pi\rangle}{\langle\pi|\bar{u} u \bar{d} d| \pi\rangle} \approx-1 .
$$

One way to estimate the penguin contributions is to consider a chiral theory in the large $N_{c}$ (the number of color) limit. It was shown in ref. 8 that the corresponding effective operator for $Q_{6}$ is

$$
-\left(\frac{f_{K}}{f_{\pi}}-1\right) \frac{4 m_{K}^{2} m_{\pi}^{2} f_{\pi}^{2}}{m_{s}^{2}} \frac{f_{\pi}^{2}}{m_{\pi}^{2}} \operatorname{Tr}\left[T^{a} \partial_{\mu} \Sigma \partial^{\mu} \Sigma^{\dagger}\right] .
$$

This contributes a term of $5.3 \times 10^{-6} \mathrm{GeV}^{4}$ to the left hand side of the second equation in (29). This is clearly too small to bring $b_{s}$ and $b_{a}$ to a same size. It is known that the large $N_{c}$ method is not always reliable ${ }^{8}$, however, for the penguin to explain the $\Delta I=1 / 2$ rule, the realistic penguin matrix element must be 40 times the large $N_{c}$ result. A recent lattice calculation shows that the large $N_{c}$ estimate is correct in order of magnitude at least for the $K \rightarrow \pi$ matrix element. ${ }^{9}$ In light of this, we take eq. (31) as qualitatively true under the assumption about the strange matrix elements.

This result, however, contradicts various valence-quark models for the pion. For instance, in the non-relativistic quark model, we have,

$$
\chi=1 / 3 .
$$

The same result can also be obtained in the vacuum insertion approximation, in which the matrix element of four-quark operators are calculated by inserting a physical vacuum in the middle. ${ }^{7}$ In the MIT bag model, we find,

$$
\chi=\frac{1}{3} \frac{\int\left(j_{0}^{4}+j_{1}^{4}-\frac{2}{3} j_{0}^{2} j_{1}^{2}\right)}{\int\left(j_{0}^{2}+j_{1}^{2}\right)^{2}},
$$

where $j_{0}$ and $j_{1}$ are upper and lower components of the bag wave function. Clearly, for any $j_{0}$ and $j_{1}, \chi$ is larger than zero, but smaller than $1 / 3$. Thus either different four-quark operators in these models acquire different renormalization constants, or the strange degrees of freedom must be added explicitly, or both.

If the above discrepancy implies a large strange content of the pion, it is the first such evidence. To support this claim, let us consider the matrix elements of a few familar strange 
quark operators in the pion. The matrix elements of type $\left\langle 0|\bar{s} \Gamma s| \pi^{0}\right\rangle$ clearly vanish due to isospin symmetry. The matrix elements of type $\left\langle\pi^{0}|\bar{s} \Gamma s| \pi^{0}\right\rangle$ vanish exactly except for $\Gamma=1$. However, the chiral symmetry predicts $\left\langle\pi^{0}|\bar{s} s| \pi^{0}\right\rangle=0$ up to high-order terms in chiral expansion. The argument goes like this: using the first-order perturbation theory we calculate the masses of Goldstone bosons, for instance, ${ }^{10}$

$$
m_{\pi}^{2}=\left\langle\pi\left|m_{u} \bar{u} u+m_{d} \bar{d} d+m_{s} \bar{s} s\right| \pi\right\rangle .
$$

On the other hand, we can calculate these masses from the axial current two-point functions,

$$
m_{\pi}^{2}=-\frac{1}{f_{\pi}^{2}}\left(m_{u}+m_{d}\right)\langle 0|\bar{u} u| 0\rangle .
$$

Matching these two results, we have

$$
\left\langle\pi^{0}|\bar{s} s| \pi^{0}\right\rangle=0 .
$$

Thus, it seems difficult to find evidence that the strange quark content is large in pion.

The $\Delta I=1 / 2$ rule is a solid experimental fact. To translate this into a statement about strange content of the nucleon and pion is not entirely straightforward. The contributions of penguin operators, particularly in the meson sector, must be calculated in a more reliable way, although they seem negligible at the scale we consider. Our analysis in the effective theory is made only at the tree order in chiral perturbation, and higher order corrections could be large despite the folklore that they are typically at the level of 30\%. Furthermore, in the pion case we do not know the real value of $\chi$ and the available methods for evaluating it do not explain the $\Delta I=1 / 2$ rule themselves. Finally, I should emphasize the scale dependence of strange content. It is possible that large strange matrix elements at the scale of $1 \mathrm{GeV}$ become negligible at the scale, say, $0.2 \mathrm{GeV}$. However, besides the untrustworthy perturbation evolution, no one knows the scale dependence of hadron matrix elements at low energy.

I thank A. Manohar and S. Sharpe for discussions on the penguin contributions. 


\section{REFERENCES}

1. J. Ashman et al., Nucl. Phys. B328 (1989) 1.

2. the SMC collaboration at CERN, the HERMES collaboration at HERA, the E142/E143 collaborations at SLAC, the SAMPLE collaboration at MIT-Bates, CEBAF proposals \#PR-91-010, \#PR-91-017, \#PR-91-004.

3. D. B. Kaplan, Phys. Lett. 275 (1992) 137.

4. J. M. Flynn and L. Randall, Nucl. Phys. B326 (1989) 31.

5. H. Georgi, Weak Interaction and Modern Particle Theory (Addison-Wesley, Reading, MA, 1984).

6. E. Jenkins, Nucl. Phys. B375 (1992) 561.

7. M. A. Shifman, A. I. Vainshtein, and V. J. Zakharov, Nucl. Phys. B120 (1977) 315.

8. R. S. Chivukula, J. M. Flynn, and H. Georgi, Phys. Lett. B171 (1986) 453.

9. S. Sharp, Nucl. Phys. (Proc. Suppl.) B20 (1990) 429.

10. J. Gasser and H. Leutwyler, Phys. Reps. 87 (1982) 77. 